\title{
Patient Reported Outcomes after Local Flap Coverage for Lower Extremity Trauma Neel Bhagat ${ }^{1}$, Scott Loewenstein ${ }^{2}$, Kevin Knox ${ }^{2}$, Joshua Adkinson², Aladdin Hassanein ${ }^{2}$, Ravinder Bamba ${ }^{2}$ \\ ${ }^{1}$ Indiana University School of Medicine, ${ }^{2}$ Indiana University Division of Plastic Surgery
}

\section{Background:}

Complex lower extremity trauma can result in devastating outcomes including amputation and poor quality of life. Limb salvage can be achieved with the use of local flaps_like muscle flaps such as the gastrocnemius or soleus flaps, which detach muscles in order to use them for local coverage. Fasciocutaneous flaps such as reverse sural and propeller flaps are other examples of local flaps which utilize overlying soft tissue for local rearrangement. Patient-reported outcome (PRO) studies have not been reported for local flaps. Additionally, the potential for functional deficits as a result of muscle flaps in comparison to fasciocutaneous flaps has not been investigated.

\section{Methods:}

Local flap coverage for lower extremity traumas between 2014-2019 were reviewed. 248 local flaps were performed for lower extremity salvage following trauma. PROs were recorded utilizing both the Lower Extremity Functional scale (LEFS) and the 36-Item Short-Form Health Survey (SF-36).

\section{Results:}

Surveys were completed by 37 patients (response rate $18.3 \%$, mean follow-up time 3.16 years, average age 49.72 years old). The average LEFS score was $42.09 \pm 14.18$, and the average physical functioning score was $43.03 \pm 21.54$. LEFS score and SF-36 physical functioning scores were significantly lower in patients who underwent muscle flaps compared to fasciocutaneous flaps ( $p=0.021$ and $p=0.022$ respectively).

\section{Conclusions and Impact:}

Patients undergoing local flaps for lower extremity reconstruction have low quality of life scores. Patients who underwent fasciocutaneous flaps had significantly higher quality of life scores compared to muscle flap patients suggesting that fasciocutaneous flaps may cause less functional deficit in salvage operations. 